Journal of Case Reports 2017;7(4):345-347

\title{
Pelvic Mass in a Post-partum Female
}

\author{
Divya Garg, Josephine Chow ${ }^{1}$
}

Department of Family Medicine, University of Calgary, Alberta, Canada; ${ }^{1}$ Department of Family Practice, University of British Columbia, Vancouver, Canada.

\author{
Corresponding Author: \\ Dr. Divya Garg \\ Email: dgarg@ucalgary.ca \\ This is an Open Access article distributed \\ under the terms of the Creative Commons \\ Attribution License (creativecommons.org/ \\ licenses/by/3.0). \\ Received \\ Accepted \\ Published

\begin{abstract}
Background: Ovarian cancer is associated with significant morbidity and mortality. It may be asymptomatic in its early phase and even when patients do seek medical attention they often present with mild and non-specific abdominal symptoms including cramping, bloating or subtle urinary symptoms. Case Report: This case presents a rare presentation of ovarian cancer in a postpartum, pre-menopausal female. Conclusion: This case illustrates the importance of having a high index of suspicion for potential ovarian cancer in patients of any age presenting with a pelvic or adnexal mass.
\end{abstract}

Keywords: Adnexal Diseases, Ovarian Neoplasms, Premenopause, Postpartum Period.

\section{Introduction}

Adnexal masses can be benign or malignant. Although ovarian cancer accounts for only three percent of cancers in women, it is associated with the highest mortality amongst gynaecological cancers $[1,2]$. Uterine cancer is most commonly diagnosed reproductive cancer but ovarian cancer has highest mortality rate with 5-year survival rate of about $44 \%$ [3-5]. Higher mortality rate is attributed to its asymptomatic presentation or absence of identifiable symptoms in its early stages [3]. Women generally present at an advanced stage with metastases and may only have vague abdominal or pelvic symptoms [1,2]. Although most commonly diagnosed in post-menopausal women, primary care providers need a high index of suspicion for considering ovarian cancer in patients of any age group presenting with an adnexal mass.

Literature and guidelines do not support screening asymptomatic women for ovarian cancer using either pelvic or trans-vaginal ultrasound or CA125 [6,7]. A systematic review and metaanalysis showed that screening asymptomatic women for ovarian cancer did not lower all cause or ovarian cancer related mortality [6]. Evidence also suggests that it does not lead to earlier diagnosis [6,7]. There is also potential harm of screening as false positives can lead to unnecessary investigations, surgery and surgical complications as well as lead to increased distress for the patient [7]. These recommendations do not apply to women with known genetic mutations that increase their risk of ovarian cancer [7]. Women with a family history of ovarian cancer or other high risk family history should be offered genetic counselling $[2,8]$.

\section{Case Report}

A 38 year old G1P1 8 month post-partum woman presented with sensation of increasing abdominal girth. At the time of her delivery, she had an urgent caesarean section for fetal distress during which a left ovarian cyst was removed. Pathology revealed a benign cystic teratoma. She presented with a one month history of increasing abdominal girth, pressure sensation, bloating and intermittent cramps. On further questioning she denied constitutional symptoms, early satiety, urinary 
symptoms or bowel symptoms. She is otherwise healthy and takes no medications aside from prenatal vitamins. Family history was unremarkable for cancer. Examination showed normal vitals. There was a palpable abdominal/midline mass one third caudally from the umbilicus. Bimanual examination revealed a non-tender large pelvic mass which was indistinguishable from the uterus. The abdomen was otherwise soft and no peritoneal signs were elicited.

A pelvic ultrasound was obtained which revealed a $9 \times 12 \times 14.5 \mathrm{~cm}$ solid and cystic mass with Doppler flow to the large solid components, consistent with a potential ovarian malignancy. Gynaecologist on call was consulted and advised further workup for risk stratification. CT chest/ abdomen/pelvis confirmed a $17 \mathrm{~cm}$ solid and cystic mass with no obvious signs of metastasis. Her hemoglobin was $115 \mathrm{~g} / \mathrm{L}$ with $\mathrm{MCV}$ of 76 fl. Tumor markers were all within normal limits, including serum $\beta$-hCG $<1 \mathrm{IU} / \mathrm{L}$ (negative), $\mathrm{LDH}$ of $241 \mathrm{U} / \mathrm{L}, \mathrm{CA} 125$ of $34.1 \mathrm{kU} / \mathrm{L}, \mathrm{AFP}$ of $2.6 \mu \mathrm{g} / \mathrm{L}$, and CA $19-9$ of $30 \mathrm{kU} / \mathrm{L}$.

She was referred urgently to gynaecologyoncology and underwent a midline laparotomy with total abdominal hysterectomy, bilateral salpingo-ophorectomy, infra-colic omentectomy, pelvic lymph node dissection, resection of right retroperitoneal mass and appendectomy. Final pathology showed clear cell carcinoma of the ovary with microscopic foci of metastasis in the retro-peritoneum and omentum with no involved nodes and no evidence of residual disease [Stage IIIA2 (pT3a2-N0/N1)]. Her post-operative course was unremarkable and she was referred to the local cancer centre for chemotherapy. Genetic testing did not identify any mutation in either BRCA1 or BRCA2 genes.

\section{Discussion}

It is important for primary care providers to consider the possibility of an underlying ovarian malignancy in patients of any age group who present with a pelvic mass. A careful history and evaluation of symptoms suggestive of ovarian cancer is crucial. Risk factors include age, smoking, nulliparity, infertility, late menopause, Ashkenazi Jewish ancestry and family history of breast or ovarian cancer $[1,2,9]$. The two most common genetic syndromes associated with ovarian cancer risk are BRCA gene mutations being involved in about $10 \%$ cases of ovarian cancer and Lynch syndrome being involved in about 2 to $3 \%$ cases of ovarian cancer $[7,10]$. Symptoms of ovarian cancer include pelvic or abdominal pain, urinary urgency or frequency, difficulties eating, early satiety, increased abdominal size, bloating and constitutional symptoms $[1,2,10]$.

A risk of malignancy index II score can be helpful in identifying patients at higher risk of ovarian cancer [1,11]. A RMI II score of over 200 is usually indicative of a higher malignancy risk [1]. However, in our patient's case this score is under 200 [Table 1]. Several tumour markers are useful in the evaluation of an ovarian mass. CA 125 is more commonly elevated in ovarian cancer but can be elevated in a number of benign and malignant conditions [2]. Elevated AFP and serum $\beta$-hCG can be indicative of a germ cell tumour [2].

Table 1: Risk of Malignancy II score

\begin{tabular}{|l|l|l|}
\hline Risk of Malignancy II score $=$ Ultrasound score $\mathrm{x}$ Menopausal score $\mathrm{x}$ CA 125 level $(\mathrm{U} / \mathrm{mL})$ & Solid areas -4 \\
\hline Ultrasound features & Multilocular cyst -1 & Postmenopausal - 4 \\
\hline Menopausal status & Premenopausal -1 & \\
\hline CA 125 level & In U/ml & - 125 level of $34.1=4 \mathrm{x} 1 \mathrm{x} 34.1=136.4$ \\
\hline
\end{tabular}




\section{Conclusion}

Primary care providers should have a high index of suspicion for potential ovarian cancer in patients presenting with pelvic or adnexal mass. Careful history should include evaluation of risk factors and symptoms suggestive of ovarian cancer. Initial workup should include a trans-vaginal or transabdominal ultrasound and consideration of tumor markers such as CA 125. If malignancy is suspected, then timely investigations and urgent referral to gynaecology oncology for optimal surgical management are important. Screening for ovarian cancer is not recommended in asymptomatic women without known high risk genetic mutations. But women with a high risk family history should be offered genetic counselling.

Contributors: DG: literature search, manuscript writing; JC: manuscript editing, literature search. DG will act as guarantor. Both authors approved final version of the manuscript.

Funding: None; Competing interests: None stated.

\section{References}

1. Le T, Giede C, Salem S, Lefebvre G, Rosen B, Bentley J, Kupets R. Initial evaluation and referral guidelines for management of pelvic/ovarian masses. Journal of Obstetrics and Gynaecology. 2009;31:668-673.

2. Glance A. Ovarian cancer: an overview. Am Fam Physician. 2009;80:609-616.
3. Navaneelan T. Trends in the incidence and mortality of female reproductive system cancers. Statistics Canada, catalogue. 2015(82-624). Available at: http://www. statcan.gc.ca/pub/82-624-x/2015001/article/14095-eng. pdf. Accessed on August 24, 2017.

4. Canadian Cancer Society's Advisory Committee on Cancer Statistics. Canadian Cancer Statistics 2016. Toronto, ON: Canadian Cancer Society. Available athttp:// www.cancer.ca/en/cancer-information/cancer-101/ canadian-cancer-statistics-publication/? region $=$ on . Accessed on August 24, 2017.

5. Permuth-Wey J, Sellers TA. Epidemiology of ovarian cancer. Methods Mol Biol. 2009;472:413-437.

6. Reade CJ, Riva JJ, Busse JW, Goldsmith CH, Elit L. Risks and benefits of screening asymptomatic women for ovarian cancer: a systematic review and meta-analysis. Gynecologic oncology. 2013;130:674-681.

7. Moyer VA. Screening for ovarian cancer: US Preventive Services Task Force reaffirmation recommendation statement. Annals of Internal Medicine. 2012;157:900904.

8. Doubeni CA, Doubeni AR, Myers AE. Diagnosis and management of ovarian cancer. Am Fam Physician. 2016;93:937-944.

9. Kaloo PL, Khazali S, Hoy D, Sadoon S. Management of Suspected Ovarian Masses in Premenopausal Women. Green-Top Guideline No. 2011;62.

10. McLemore MR, Miaskowski C, Aouizerat BE, Chen LM, Dodd MJ. Epidemiologic and genetic factors associated with ovarian cancer. Cancer nursing. 2009;32:281.

11. Morgante G, Marca A, Ditto A, Leo V. Comparison of two malignancy risk indices based on serum CA125, ultrasound score and menopausal status in the diagnosis of ovarian masses. BJOG. 1999;106:524-527. 